\title{
Novel Quality Measure and Efficient Resolution of Convex Hull Pricing for Unit Commitment
}

This paper was downloaded from TechRxiv (https://www.techrxiv.org).

\section{LICENSE}

CC BY 4.0

SUBMISSION DATE / POSTED DATE

02-03-2022 / 03-03-2022

\section{CITATION}

Bragin, Mikhail; Luh, Peter; Yan, Bing; Zheng, Tongxin; Schiro, Dane A.; Zhao, Feng; et al. (2022): Novel Quality Measure and Efficient Resolution of Convex Hull Pricing for Unit Commitment. TechRxiv. Preprint. https://doi.org/10.36227/techrxiv.19294244.v1

DOI

10.36227/techrxiv.19294244.v1 


\title{
Novel Quality Measure and Efficient Resolution of Convex Hull Pricing for Unit Commitment
}

\author{
Mikhail A. Bragin, ${ }^{*}$ Member, IEEE, Bing Yan, ${ }^{*}$ Member, IEEE, Peter B. Luh, Life Fellow, IEEE, Tongxin Zheng, Senior \\ Member, IEEE, Dane A. Schiro, Member, IEEE, Feng Zhao, Member, IEEE, and Jinye Zhao, Member, IEEE
}

\begin{abstract}
Electricity prices determined from economic dispatch without considering fixed costs may cause high uplift payments. With fixed costs, however, a price is not a monotonic function of demand, affecting market transparency. To overcome these, convex hull $(\mathrm{CH})$ pricing has recently been introduced for unit commitment with fixed costs. Several CH pricing methods were presented, and a feasible cost was used to quantify the $\mathrm{CH}$ price quality. The associated difficulties are 1 . high computational effort required to obtain a feasible cost and 2. the associated duality gap may not be tight enough to provide accurate measure. In this paper, a novel measure to quantify the quality of $\mathrm{CH}$ prices is presented by establishing an upper bound to the optimal dual value approaching it from above. Near-optimal $\mathrm{CH}$ prices are efficiently obtained by using Surrogate Lagrangian Relaxation (SLR), meanwhile, the upper bound decreases fast due to convergence of SLR. Testing results on the IEEE 118-bus system without transmission capacities indicate that the novel quality measure reaches a value of less than $0.1 \%$ in seconds - much more accurate and faster than the measure provided by a feasible cost - demonstrating the high quality of the upper bound and the efficiency of SLR. Index Terms - Electricity Markets, Convex Hull Pricing, Surrogate Lagrangian Relaxation
\end{abstract}

\section{INTRODUCTION}

In the US electricity markets, prices are usually determined based on the economic dispatch (ED) problem [1]. Since commitment costs (e.g., start-up and no-load costs) are not considered, uplift payments ${ }^{1}$ could be high. If prices are determined based on the unit commitment (UC) problem with such costs considered, the commitment-related binary variables lead to non-convexity. Consequently, prices based on a UC problem are non-monotonic with respect to demand [2], thereby affecting the transparency of the markets.

To address the above issues, convex hull $(\mathrm{CH})$ pricing has been introduced in [2]. One of the approaches to obtain $\mathrm{CH}$ prices is to solve the dual problem of a UC problem [3]-[4]. Accordingly, the $\mathrm{CH}$ prices are the optimal Lagrangian multipliers $(\lambda)$, and the uplift payment is the difference between a feasible cost to the UC problem and a dual value of the corresponding dual problem. The difficulty of this approach comes down to the multiplier-convergence issue of the standard subgradient methods and multiplier zigzagging [5]. While

\footnotetext{
This work was supported in part by National Science Foundation under Grants ECCS-1810108, and in part by a project funded by ISO New England.

M. A. Bragin $(\bowtie)$ and P. B. Luh are with the Department of Electrical and Computer Engineering, University of Connecticut, Storrs, CT 06269 USA (email: mikhail.bragin(aconn.edu).

B. Yan is with the Department of Electrical and Microelectronic Engineering,
}

several $\mathrm{CH}$ pricing solution methods have been presented, a feasible cost [6] was used to quantify the quality of these prices, i.e., how close the prices are to the optimal prices. The associated difficulties with the current quality-measuring approach is 1 . high computational effort required to obtain a feasible cost and 2 . the associated duality gap may not be tight enough to provide high quality measure as schematically demonstrated in Figure 1 below. Therefore an alternative tighter measure (upper bound) is desired.

$q(\lambda)$

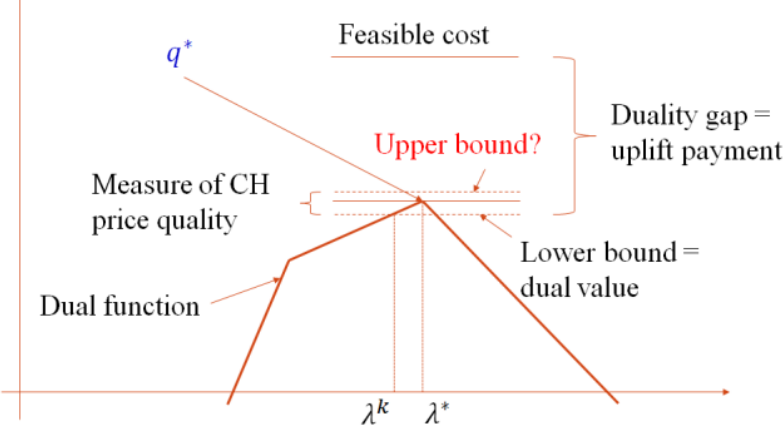

Fig 1: Dual function and the novel measure of $\mathrm{CH}$ price quality.

To overcome these difficulties, the goal of this paper is to provide a high-quality measure of $\mathrm{CH}$ prices through the development of a novel upper bound to the optimal dual value, and to obtain $\mathrm{CH}$ prices computationally efficiently.

After briefly presenting the UC problem formulation in Section II, a novel quality measure is developed to quantify the quality of the resulting $\mathrm{CH}$ prices in Section III. It is based on the difference between the innovative upper bound $\bar{q}$ and the best available dual value $q\left(\lambda^{k}\right)$ as shown in Figure 1. The nearoptimal $\mathrm{CH}$ prices are obtained by using the Surrogate Lagrangian Relaxation (SLR) method [7] which overcame the difficulties of standard subgradient methods. Meanwhile, fast convergence of SLR leads to fast decrease of the upper bound as well. Testing results based on the IEEE 118-bus system without transmission capacity constraints indicate that the $\mathrm{CH}$ price quality-measure of $<0.1 \%$ is reached in seconds - much more accurate and faster as compared to the measure that a feasible cost can provide - demonstrating both the high quality of the upper bound and the efficiency of the SLR method.

\section{UC PROBLEM FOR CONVEX HULL PRICING}

The purpose of this section is to present a simplified formulation of the UC problem to lay the foundation of the

Rochester Institute of Technology, Rochester, NY 14623 USA.

*Equal contribution

T. Zheng, D. A. Schiro, F. Zhao, J. Zhao, and I. Lelic are with the Advanced Technology and Solutions, ISO New England, Holyoke, MA 01040 USA.

${ }^{1}$ Uplift payments are shortfalls between the energy payments from customers and the necessary payments for units. 
further presentation of $\mathrm{CH}$ prices as well as for the development of the novel quality measure for $\mathrm{CH}$ prices. Consider a UC problem with $I$ units indexed by $i$ and $T$ hours indexed by $t$. For unit $i$, decision variables at time $t$ are: binary commitment $x_{i, t}$ and startup $u_{i, t}$ as well as continuous dispatch $p_{i, t}$. For simplicity, ancillary services and transmission capacity constraints are not considered following [8]. System-wide constraints only include system demand. Unit-level constraints include generation capacity, start-up, minimum up and down time, and ramp rate.

(1) Objective function: The objective is to minimize the total generation, start-up and no-load cost as:

$$
\min _{\left\{x_{i}, u_{i}, p_{i}\right\}}\left\{\sum_{i=1}^{I} z_{i}\left(x_{i}, u_{i}, p_{i}\right)\right\} \text {, }
$$

with $z_{i}\left(x_{i}, u_{i}, p_{i}\right)=\sum_{t=1}^{T}\left(f_{i}\left(p_{i, t}\right)+c_{i}^{S} \cdot u_{i, t}+c_{i}^{N} \cdot x_{i, t}\right)$ as the total cost of unit $i$ consisting of energy costs and fixed costs, where $f_{i}$ is a monotonic piecewise linear cost function, $c_{i}^{S}$ is a start-up cost and $c_{i}^{N}$ is a no-load cost.

(2) System demand constraint: The total power generation equals the demand at each time $t$ :

$$
\sum_{i=1}^{I} p_{i, t}=D_{t}, t=1, \ldots, T,
$$

where $D_{t}$ is the demand at time $t$.

(3) Unit-level constraints

1) Generation capacity: When unit $i$ is online, the generation levels $p_{i, t}$ are between minimum $P_{i}^{\text {min }}$ and maximum $P_{i}^{\text {max }}$ :

$$
x_{i, t} \cdot P_{i}^{\min } \leq p_{i, t} \leq x_{i, t} \cdot P_{i}^{\max } .
$$

2) Start-up: The binary start-up status is captured through binary variables $u_{i, t}$ as:

$$
x_{i, t}-x_{i, t-1} \leq u_{i, t}
$$

3) Minimum up and down time: Unit $i$ stays online (offline) for its minimum up (down) time, i.e.,

$$
\sum_{j=t-L_{i}+1}^{t} u_{i, j} \leq x_{i, t}, \sum_{j=t-l_{i}+1}^{t} u_{i, j} \leq 1-x_{i, t-l_{i}},
$$

where $l_{i}$ and $L_{i}$ are the minimum up- and down-time of unit $i$.

4) Ramp rate: The change of generation levels between two consecutive hours cannot exceed its ramp-rate requirements:

$$
\begin{gathered}
p_{i, t}-p_{i, t-1} \leq R_{i} \cdot x_{i, t-1}+V_{i} \cdot\left(1-x_{i, t-1}\right), \\
p_{i, t-1}-p_{i, t} \leq R_{i} \cdot x_{i, t}+V_{i} \cdot\left(1-x_{i, t}\right),
\end{gathered}
$$

where $R_{i}$ is the ramp-up/down rate and $V_{i}$ is the start-up/shutdown ramp rate.

\section{CONVEX Hull Pricing Through the DuAl Problem}

This section is on the development of the novel measure to quantify the quality of the $\mathrm{CH}$ prices. After briefly presenting a dual problem in subsection $A$, the novel measure will be developed in subsection $\mathrm{B}$.

\section{A. The dual problem of the UC problem}

The dual problem to the UC problem is defined as [8]:

$\max _{\lambda} q(\lambda)$,

where $q(\lambda)$ is the dual function defined as:

$$
q(\lambda)=\min _{\{p, x, u\} \in \mathscr{F}} L(\lambda, p, x, u) .
$$

Here $\mathfrak{F}$ is a feasible set delineated by generation capacity (3), start-up (4), minimum up- and down-time (5), as well as ramp- rate constraints (6)-(7), and $L(\lambda, p, x, u)$ is the Lagrangian function defined as:

$$
L(\lambda, x, u, p)=\sum_{i=1}^{I} z_{i}\left(x_{i}, u_{i}, p_{i}\right)+\sum_{t=1}^{T} \lambda_{t}\left(\sum_{i=1}^{I} p_{i, t}-D_{t}\right),
$$

where $\lambda=\left\{\lambda_{t}\right\}$ is a $1 \times T$ vector of Lagrangian multipliers relaxing system-wide demand constraints (2). The $\mathrm{CH}$ prices are optimal multipliers $\lambda^{*}$ of the dual problem (8) [2].

\section{B. Novel quality measure for convex hull pricing}

This subsection is on the novel way to obtain the upper bound $\bar{q}$ to the optimal dual value $q\left(\lambda^{*}\right)$ approaching $q\left(\lambda^{*}\right)$ from above. Together with the lower bound, i.e., a dual value $q\left(\lambda^{k}\right)$ at iteration $k$, the novel quality measure for $\mathrm{CH}$ prices will be obtained as a relative difference between $\bar{q}$ and $q\left(\lambda^{k}\right)$.

The main premise behind the derivation of the upper bound is the fact proved in [9, pp. 704-706] stating that if the following condition on stepsizes holds

$$
s^{k}<\frac{q^{*}-\tilde{L}\left(\lambda^{k}, x^{k}, u^{k}, p^{k}\right)}{\left\|\tilde{g}\left(p^{k}\right)\right\|^{2}},
$$

where $\tilde{L}\left(\lambda^{k}, x^{k}, u^{k}, p^{k}\right)$ is the so-called Lagrangian function, with tilde indicating that the function (10) is minimized with respect to one of few subproblems at a time, then multipliers approach $\lambda^{*}$ in the following way:

$$
\left\|\lambda^{*}-\lambda^{k+1}\right\|^{2}<\left\|\lambda^{*}-\lambda^{k}\right\|^{2}
$$

A direct consequence of the above is that if multipliers move away from $\lambda^{*}$, i.e.,

$$
\left\|\lambda^{*}-\lambda^{k+1}\right\|^{2}>\left\|\lambda^{*}-\lambda^{k}\right\|^{2}
$$

then it could only be a consequence of the violation of stepsizing formula (11), i.e.,

$$
s^{k} \geq \frac{q^{*}-\tilde{L}\left(\lambda^{k}, x^{k}, u^{k}, p^{k}\right)}{\left\|\tilde{g}\left(p^{k}\right)\right\|^{2}} .
$$

According to (14), there exists an overestimate of the optimal dual value $\bar{q}>q^{*}$ such that

$$
s^{k}=\frac{\bar{q}-\tilde{L}\left(\lambda^{k}, x^{k}, u^{k}, p^{k}\right)}{\left\|\tilde{g}\left(p^{k}\right)\right\|^{2}} .
$$

From (15), the overestimate can be expressed as:

$$
\bar{q}=s^{k}\left\|\tilde{g}\left(p^{k}\right)\right\|^{2}+\tilde{L}\left(\lambda^{k}, x^{k}, u^{k}, p^{k}\right) .
$$

However, the difficulty is that $\lambda^{*}$ is not known and the upper bound (16) cannot be computed.

To resolve this difficulty, our main idea is as follows: if multipliers do not approach any common value (including $\lambda^{*}$ ) during several iterations, then conditions (14)-(16) hold. To determine whether multipliers approach any common value or not, the following "auxiliary feasibility" problem is solved:

$$
\begin{aligned}
& \left\|\lambda-\lambda^{k+1}\right\|^{2} \leq\left\|\lambda-\lambda^{k}\right\|^{2}, \\
& \left\|\lambda-\lambda^{k+2}\right\|^{2} \leq\left\|\lambda-\lambda^{k+1}\right\|^{2}, \\
& \ldots \\
& \left\|\lambda-\lambda^{k+n}\right\|^{2} \leq\left\|\lambda-\lambda^{k+n-1}\right\|^{2} .
\end{aligned}
$$

With respect to (17), $\lambda$ is a $1 \times T$ vector of continuous decision variables (not to be confused with dual variables within (8)).

The problem (17) starts from $k=1, n=1$. After multipliers are updated, $n$ is increased by 1 until (17) admits no feasible solution. Since at one of the iterations, equality (14) holds, the sought-for upper bound is then obtained as:

$$
\bar{q}=\max _{\kappa \in[k+1, k+n]}\left\{s^{\kappa} \cdot\left\|\tilde{g}\left(p^{\kappa}\right)\right\|^{2}+\tilde{L}\left(\lambda^{\kappa}, x^{\kappa}, u^{\kappa}, p^{\kappa}\right)\right\}
$$

Subsequently, values of $k$ and $n$ are reset as $k \rightarrow k+n, n \rightarrow 1$, and the procedure repeats. 
Finally, the quality measure of the $\mathrm{CH}$ prices is defined as $\frac{\bar{q}^{k}-q^{k}}{\bar{q}^{k}}$, where $\bar{q}^{k}$ is the best (lowest) upper bound, and $q^{k}$ is the best (highest) dual value obtained up to iteration $k$. This measure can be used as a stopping criterion.

Unlike feasible costs, the novel upper bound approaches the optimal dual value from above since the upper bound (18) depends on the stepsizes approaching zero and the Lagrangian function approaching the optimal dual value as proved in [7]. Another implication of the above, fast convergence of SLR leads to fast convergence of the upper bound to the dual value.

\section{NumericAl Testing}

This section is to demonstrate the quality of the novel measure of $\mathrm{CH}$ prices. In Example 1, a small 2-unit, 10-hour UC problem is considered. In Example 2, a UC problem based on the IEEE 118-bus system without transmission capacity constraints is considered. The method is implemented using IBM ILOG CPLEX Optimization Studio V 12.10.0.0 on a PC with $2.40 \mathrm{GHz}$ Intel ${ }^{\circledR}$ Xeon ${ }^{\circledR}$ E-2286M CPU and 32G RAM.

Example 1. Consider a UC problem with two units and a time horizon of 10 hours. Unit characteristic are summarised below: Unit 1: $P^{\min }=50 \mathrm{MW}, P^{\max }=200 \mathrm{MW}$, ramp up/down rate $R=$ $200.6 \mathrm{MW} / \mathrm{hr}$, start-up/shut-down ramp rate $V=150.3 \mathrm{MW} / \mathrm{hr}$, generation cost $=65 \$ / \mathrm{MW}$, and start-up cost $c^{S}=\$ 0$;

Unit 2: $P^{\min }=50 \mathrm{MW}, P^{\max }=200 \mathrm{MW}$, ramp-up/down rate $R=$ $40.7 \mathrm{MW} / \mathrm{hr}$, start-up/shut-down ramp rate $V=70.35 \mathrm{MW} / \mathrm{hr}$, generation cost $=40 \$ / \mathrm{MW}$, and start-up cost $c^{S}=\$ 6000$.

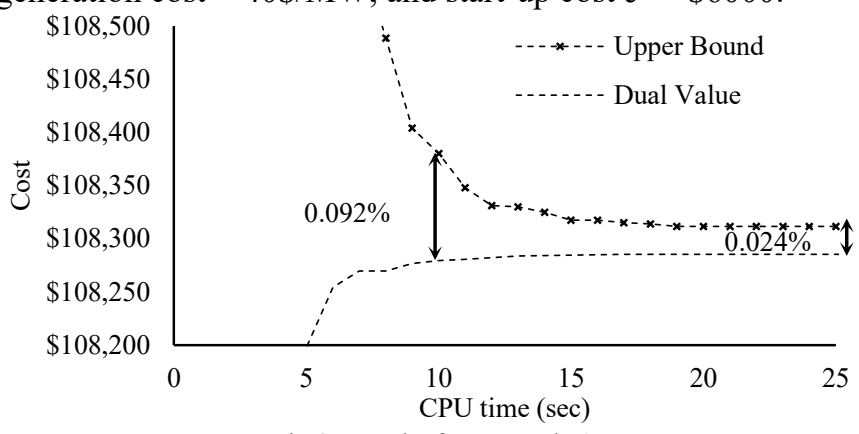

Fig 2: Results for Example 1

The results obtained by using SLR are shown in Figure 2 above. It can be seen that near-optimal $\mathrm{CH}$ prices with quality less than $0.1 \%$ are obtained in 10 seconds (sec). The CPU time includes subproblem solving (99.1\%), multipliers updating $(0.76 \%)$, and upper bound searching $(0.14 \%)$, demonstrating that the effort involved in calculating the upper bound is very minor, unlike that required to obtain feasible costs. Compared to the standard duality gap of $1.8 \%$ for this instance, the novel upper bound of $0.024 \%$ is roughly 75 times more accurate.

Example 2. Consider a 54-unit, 24-hour UC problem based on the IEEE 118-bus system.

The results obtained by using SLR are shown in Figure 3. With the the novel upper bound, the gap of $0.038 \%$ is obtained in $32.33 \mathrm{sec}$, with subproblem solving time of $31.48 \mathrm{sec}$ (97.37\%), multipliers updating time of $0.59 \mathrm{sec}(1.825 \%)$, and upper bound searching time of $0.26 \mathrm{sec}(0.805 \%)$. What is more, there are several observations:

1. The quality of $\mathrm{CH}$ prices is comparable to that obtained for a much smaller problem of Example 1, in terms of both CPU time and quality.

2. Compared to the problem-dependent standard duality gap
$(0.09 \%)$, the novel quality measure $(0.038 \%)$ is problemindependent, and it is roughly 2.4 times more accurate.

3. Computationally, the standard quality gap requires a feasible solution, which for this problem is obtained in $0.504 \mathrm{sec}$, roughly 2 times longer than to obtain the novel upper bound. When the number of units increases, the feasible solution finding time is expected to increase. The CPU time required to compute the novel quality measure is not expected to increase for larger-scale problems as calcualting upper bounds only requires 24 decision variables within (17), paving the way for providing a tight measure of $\mathrm{CH}$ prices for large-scale problems as a modest CPU time.

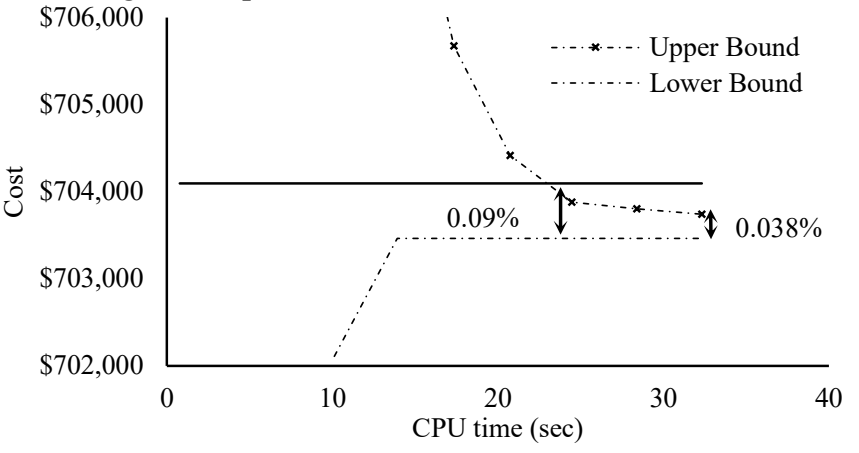

Fig 3: Results for Example 2

V. CONCLUSION

In this paper, a novel measure for $\mathrm{CH}$ prices is developed and the near-optimal $\mathrm{CH}$ prices are efficiently obtained by using our recent SLR. Through numerical testing, it is demonstrated that the near-optimal $\mathrm{CH}$ prices with high quality are obtained fast and the computational effort involved in obtaining an upper bound for the quality measure is in seconds, less than $1 \%$ of the total time. The method to quantify the quality of $\mathrm{CH}$ prices is generic within the LR-based methods and can be generally used to quantify the quality of the dual solutions.

\section{REFERENCES}

[1] E. Litvinov, F. Zhao and T. Zheng, "Electricity Markets in the United States: Power Industry Restructuring Processes for the Present and Future," IEEE Power and Energy Magazine, vol. 17, no. 1, 32-42, 2019.

[2] P. Gribik, W. Hogan, and S. Pope, "Market-clearing electricity prices and energy uplift," Harvard University, Cambridge, MA, USA, working paper, 2007.

[3] C. Wang, T. Peng, P. B. Luh, P. Gribik, and L. Zhang, "The Subgradient Simplex Cutting Plane Method for Extended Locational Marginal Prices," IEEE Transactions on Power Systems, vol. 28, no. 3, 2758-2767, 2013.

[4] G. Wang, U. V. Shanbhag, T. Zheng, E. Litvinov, and S. Meyn, "An Extreme-Point Subdifferential Method for Convex Hull Pricing in Energy and Reserve Markets - Part I: Algorithm Structure," IEEE Transactions on Power Systems, vol. 28, no. 3, 2111-2120, 2013.

[5] P. B. Luh, D. Zhang, and R. N. Tomastik, "An algorithm for solving the dual problem of hydrothermal scheduling," IEEE Trans. Power Syst., vol. 13, no. 2, 593-600, 1998.

[6] Y. Yu, T. Zhang, Y. Guan, and Y. Chen. "Convex primal formulations for convex hull pricing with reserve commitments," IEEE Transactions on Power Systems, vol. 36, no. 3, 2345-2354, 2020.

[7] M. A. Bragin, P. B. Luh, J. H. Yan, N. Yu and G. A. Stern, "Convergence of the Surrogate Lagrangian Relaxation Method," J. Optim. Theory Appl., vol. 164 , no. 1, 173-201, 2015.

[8] B. Hua and R. Baldick, "A convex primal formulation for convex hull pricing," IEEE Trans. Power Syst., vol. 32, no. 5, 3814-3823, 2017, doi: 10.1109/TPWRS.2016.2637718.

[9] X. Zhao, P. B. Luh, and J. Wang, "Surrogate gradient algorithm for Lagrangian relaxation," J. Optim. Theory Appl., vol. 100(3), 699-712, 1999. 